\title{
The Effect on Day-Long Glycemia of Consuming Lower and Higher Glycemic Index Diets in People with Type 2 Diabetes: A Randomized Crossover Study
}

AN Reynolds*, Tekinkaya $\mathrm{H}$ and BJ Venn

University of Otago, Dunedin, New Zealand

\begin{abstract}
Objective: Treatment of type 2 diabetes includes pharmacologic and lifestyle modification such as dietary change. The use of the Glycemic Index (GI) to guide food choice has been advocated, although the effectiveness of this dietary strategy in people with type 2 diabetes has had mixed success. Our objective was to investigate daylong glycemic responses to diets differing in $\mathrm{Gl}$ using continuous glucose monitoring in people with type 2 diabetes.

Methods: A randomized crossover trial in 22 adults aged 18 to 75 years diagnosed with type 2 diabetes and without major co-morbidities. Lower and higher GI diets were consumed over a five-day period with food supplied to participants. Diet and physical activity were standardized and medication was maintained for the study period. Main outcomes using a Continuous Glucose Monitoring System (CGMS) were mean 24-hour glucose, three-hour incremental postprandial glycemia (iAUC), total day-long glycemia (AUC), and 48-hour glycemic variability assessed as Mean Amplitude of Glycemic Excursion (MAGE)
\end{abstract}

Results: Complete CGMS data for 18 participants were obtained. The between-treatment difference in GI was $13 \mathrm{GI}$ units $(P<0.01)$. Between low- and high-GI diets, no difference in three-hour iAUC $(\mathrm{mmol} / \mathrm{L} \cdot \mathrm{min})$ following breakfast ( 367 vs. $390, P=0.69$ ), lunch ( 252 vs. $317, P=0.16)$ or dinner $(216$ vs. $263, P=0.32)$ was observed. No difference in mean 24 -hour glucose $(6.62 \mathrm{vs} .6 .31 \mathrm{mmol} / \mathrm{L}, \mathrm{P}=0.31)$, total day-long glycemia $(8,906 \mathrm{vs} .8,786 \mathrm{mmol} /$ $L \cdot \min , P=0.82$ ) or MAGE (3.7 vs. $3.9 \mathrm{mmol} / \mathrm{L}, \mathrm{P}=0.61$ ) were observed between diets.

Conclusions: Differences in dietary GI were not predictive of improvement in within-day markers of glycemic control in people with type 2 diabetes. These findings are reflective of ongoing difficulties in translating laboratorygenerated GI values of individual foods to glycemic improvement in the whole of diet setting.

Keywords: Diabetes; Glycemic index; Continuous glucose monitoring; Glycemic control

\section{Background}

Effective treatment of type 2 diabetes mellitus entails pharmacologic and lifestyle modification. Efforts to establish standard treatments for people with type 2 diabetes are complicated by genetic and physiologic heterogeneity [1,2]. Lifestyle recommendations for people with type 2 diabetes are to undertake regular physical activity and to learn to eat well [3]. Dietary recommendations include targeting carbohydrate intake given the glycemic raising capability of such foods. Guidance is usually given regarding both quantity and type of carbohydrate with 'type' referring to the glycemic-elevating profile of food, most often expressed as the Glycemic Index (GI). For a given amount of carbohydrate, a high GI food is characterized as inducing a large and rapid rise in blood glucose concentration whereas a low GI food generates a smaller more gradual change [4]. These differences are most apparent under GI testing conditions in which individual foods containing a standard amount of carbohydrate are compared. It is unclear how useful this guidance for food selection may be in a practical setting in which foods are eaten in various amounts as part of meals [5]. The effect of manipulating the GI of diets has been tested in people with diabetes using glycated hemoglobin (HbA1C) as a primary indicator of glycemic control. In people with diabetes, raised $\mathrm{HbA1C}$ is associated with increased diabetic complications making it a useful outcome measure by which to assess treatment effect [6]. In a randomized controlled trial lasting six months, Jenkins and colleagues compared differences in HbAlC between groups of people with type 2 diabetes consuming either a low GI diet or a high cereal fiber diet [7]. A difference in HbA1C between groups of $0.18 \%$ (95\% CI $0.07,0.29)$ favoring the low GI group was found. In another trial from this research group, a low GI diet achieved through the incorporation of legumes was compared over three months with a high cereal fiber diet in people with type 2 diabetes, again with a difference in $\mathrm{HbA1C}$ of $0.2 \%$ (95\% CI $0.1,0.3$ ) favoring the low GI diet [8]. Wolever and colleagues compared three diets, namely low GI, high GI, and low glycemic load, consumed by people with type 2 diabetes for 12 months [9]. In that study, there was no differential effect of diet on HbA1C among the groups, with all three groups showing an increase over the year. Thus, although there is evidence to suggest some benefit to people with type 2 diabetes following a low GI diet, Jenkins and colleagues described the effect on HbA1C as 'moderate' while Wolever et al. observed none [7,9]. Given that GI influences postprandial glycemia over the short term, it might be expected that the acute glycemic benefits of low GI foods would accumulate over a period long enough to influence $\mathrm{HbA1C}$. The reason that $\mathrm{HbA1C}$ is relatively resilient to change in dietary GI has not been studied with the intervention effect less than expected in well-designed randomized controlled trials. It would be useful to gain insight into the day-long glycemic profiles of people with type 2 diabetes following low and high

*Corresponding author: Bernard J Venn, PhD, University of Otago, Dunedin, New Zealand, Tel: +6434795068; E-mail: bernard.venn@otago.ac.nz

Received June 24, 2014; Accepted September 18, 2014; Published September 25, 2014

Citation: Reynolds AN, Tekinkaya H, Venn BJ (2014) The Effect on Day-Long Glycemia of Consuming Lower and Higher Glycemic Index Diets in People with Type 2 Diabetes: A Randomized Crossover Study. J Diabetes Metab 5: 436 doi:10.4172/2155-6156.1000436

Copyright: (C) 2014 Reynolds AN, et al. This is an open-access article distributed under the terms of the Creative Commons Attribution License, which permits unrestricted use, distribution, and reproduction in any medium, provided the original author and source are credited. 
Citation: Reynolds AN, Tekinkaya H, Venn BJ (2014) The Effect on Day-Long Glycemia of Consuming Lower and Higher Glycemic Index Diets in People with Type 2 Diabetes: A Randomized Crossover Study. J Diabetes Metab 5: 436 doi:10.4172/2155-6156.1000436

Page 2 of 5

GI diets. Our hypothesis is that there would be a difference in withinday glucose markers for people with type 2 diabetes consuming low and high GI diets. This is to be achieved using a Continuous Glucose Monitoring System (CGMS) to assess the glycemic impact of foods in whole diets over two complete 48 hour periods [10,11]. Our objective is to quantify postprandial and day-long glycemia using CGMS in freeliving people with type 2 diabetes consuming diets of higher and lower GI.

\section{Research Design and Methods}

Twenty two adults (12 women and 10 men) diagnosed with type 2 diabetes were recruited through newspaper advertisement and from a Diabetes Clinic (Diabetes Otago) over a six month period at the end of 2012. Study participants were adults aged 18-75 years diagnosed with type 2 diabetes. Exclusion criteria were insulin dependence, diagnosed cardiovascular disease, pregnancy or lactation. All study participants provided written consent before commencing, the study design received approval from the University of Otago human ethics committee.

Using published peer reviewed data for CGMS output in people with type 2 diabetes, it was estimated that a crossover design of 18 participants would be sufficient to detect a practically achievable and clinically significant difference of $1 \mathrm{mmol} / \mathrm{L}$ in mean glucose readings taken over 24 hours with $85 \%$ power and a significance level of 0.05 [12].

\section{Study design}

The study was conducted over five days during which four complete 24 hour periods of glucose measures were captured with CGMS devices. Treatment involved participants consuming high or low GI meals for two consecutive 24 hour periods followed by the alternative treatment such that duplicate 24 hour periods on each diet were captured by CGMS. The order of treatment was randomized to each participant against a computer-generated process implemented before study commencement, with no washout between treatments. Participants were instructed to maintain their usual medication and level of physical activity. Demographic and anthropometric data were collected at baseline. The flow of participants through the trial is displayed in Figure 1.

\section{Diets and diet records}

Breakfast, lunch and dinner foods were provided to participants. For foods that had been tested for GI, the values were taken directly from manufacturer information or from published data [13]. The evening meals were frozen dinners that had not been tested for GI, hence the GI of these meals, and of the diets themselves, were calculated using a recognized GI composite formula [14]. The low GI foods were a ready-to-eat breakfast cereal (Kellogg's Australia Special K; GI 54), whole grain bread (Vogels Soy and Linseed; GI 49) and a frozen pasta, chicken and vegetable meal (Wattie's Chicken Penne; GI 41). High GI foods were wheat biscuits (Sanitarium Weetbix; GI 69), white bread (Nature's Fresh white bread; GI 75) and a frozen rice, chicken and vegetable meal (Wattie's Honey and Soy Chicken; GI 68). To ensure the amount of available carbohydrate was comparable between diets, the bread and frozen meals were chosen to provide similar carbohydrate per serve, and participants were instructed to weigh a portion of Special K corresponding to the carbohydrates contained in the number of wheat biscuits they consumed. Snack foods were not provided to allow a small degree of autonomy in participant diet. If consumed, participants were encouraged to standardize snack foods across the

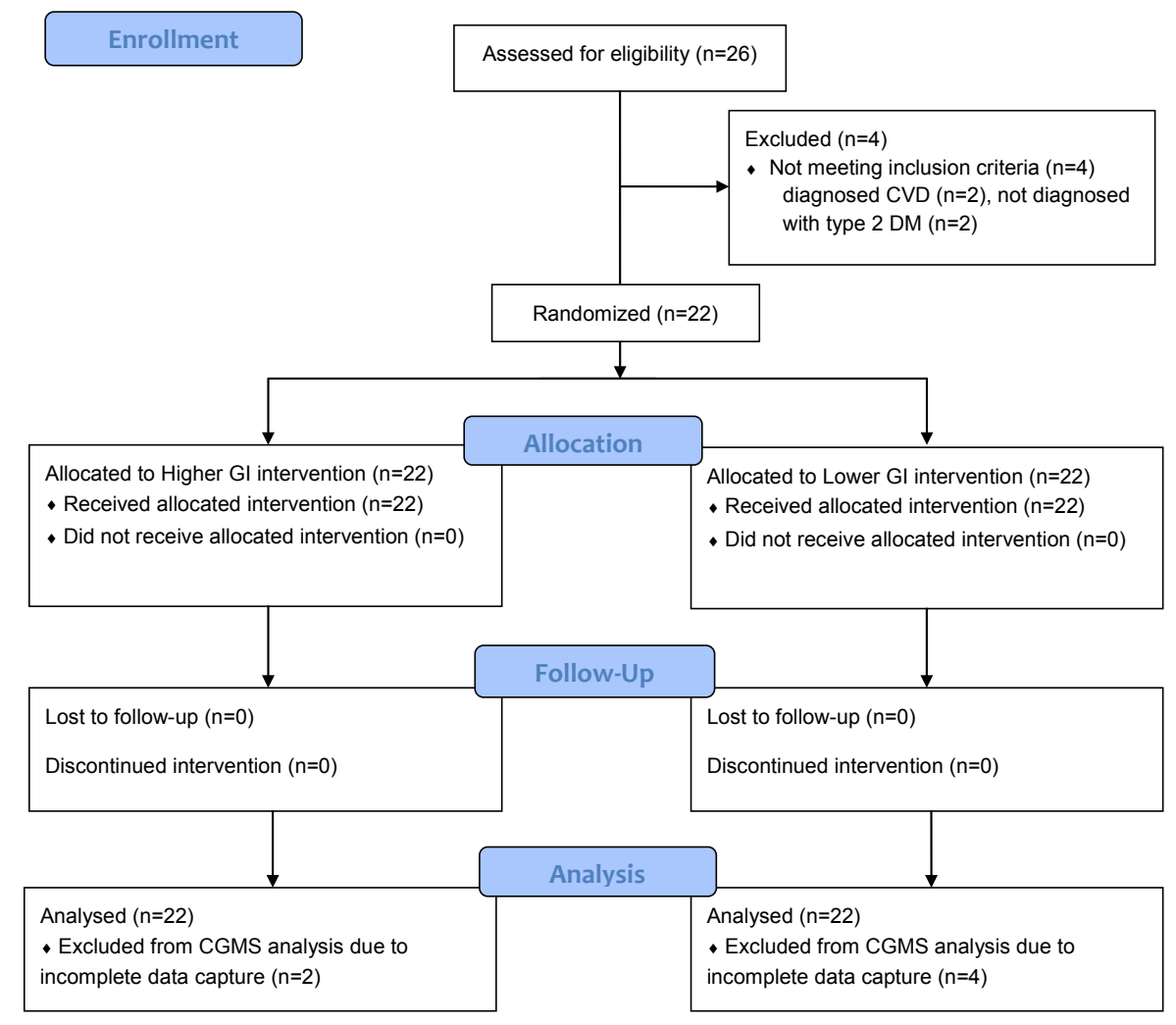

Figure 1: Flow Diagram. 
Citation: Reynolds AN, Tekinkaya H, Venn BJ (2014) The Effect on Day-Long Glycemia of Consuming Lower and Higher Glycemic Index Diets in People with Type 2 Diabetes: A Randomized Crossover Study. J Diabetes Metab 5: 436 doi:10.4172/2155-6156.1000436

Page 3 of 5

study period. Participants were blinded to the randomization process, remaining uninformed of the intervention order for the study duration. Participants were provided with electronic kitchen scales reading to the nearest gram (Salter Housewares Limited, Tonbridge, England) and received training by a nutritionist in the correct use of the scales and to complete a food diary for the trial period. The completed diet records were reviewed with the participants when they returned to have the CGMS device removed with all notation queries resolved. Dietary data were assessed using New Zealand Food Composition Files and inhouse software (Kaiculator, University of Otago, Dunedin, NZ) [15]. The nutrient content of all foods consumed were present in the Food Composition Files with the exception of the frozen meals. The nutrient composition of these meals were extracted from the meals Nutrition Information Panels and added into the dietary analysis package.

\section{Physical activity}

The nature and duration of physical activity other than light office duties were recorded for the duration of the study. Participants were instructed to standardize their physical activity across the study period.

\section{Glucose monitoring}

The continuous glucose monitor system (Medtronic iPro2) comprises a wire sensor inserted into abdominal subcutaneous tissue connected to a blinded data recorder. The small device can be worn during normal daily activities including bathing/showering and sleeping. The CGMS devices were attached peri-abdominally and allowed to imbed before recording interstitial fluid glucose concentration every 5 minutes for the duration of the trial. CGMS readings were calibrated against four self-administered capillary blood readings within every 24 hour period using meters and test strips (Free Style Optimum) that were recorded in the participant's daily diary. To retrieve the CGMS data, the sensor information and capillary data were uploaded to the Medtronic website. The sensors were attached on a Monday morning and removed on the evening of the following Friday. Participants recorded the start time of the evening meal on Monday and the sensor reading at that time was taken as the pre-prandial baseline for day one. For Tuesday to Friday, the eight-hour overnight average preceding breakfast on each of those days was taken as the baseline glucose concentration for each subsequent 24 hour period.

\section{Outcome measures}

The primary outcome measures were average glucose readings taken over 24 hours and incremental area-under-the-curve glucose concentrations (iAUC) over three hours following each meal (breakfast, lunch and dinner) for the duration of the study period. Secondary measures were total AUC over each 24 hour period and a measure of glycemic variability, represented by the Mean Amplitude of Glycemic Excursion (MAGE) [16].

\section{Data handling and statistical analysis}

AUC were calculated by the trapezoidal method using a program written for Microsoft Excel (Microsoft Corporation, Redmond, WA). Statistical analyses were carried out using a software package (Stata; release 10. StataCorp LP, College Station TX). MAGE were calculated using a computer algorithm [17]. Student's two-tailed t tests were used to test for differences in meal composition between high and low GI diets and the outcome measures.

\section{Results}

Characteristics of the 22 participants are given in Table 1. CGMS data from two participants were incomplete or unusable due to unintended removal of the device, leaving data from 20 participants to be included in the analysis of the higher GI diets. Two further CGMS data sets were excluded from the lower GI diet period due to intermittent data capture or low compliance with the required capillary calibration. The majority of participants were of European descent.

The overall composition of the lower and higher GI diets as calculated from the weighed food records are given in Table 2. According to diet records, participants displayed complete compliance in consuming the provided foods.

There was no difference in average duration of daily activity between test periods, $103.3 \mathrm{~min}(95 \% \mathrm{CI}: 101.2,105.3)$ and $104.9 \mathrm{~min}(95 \% \mathrm{CI}$ $103.0,106.8)$ on low and high GI days respectively $(\mathrm{P}>0.05)$. No glucose response to any of the very light and light exercise was observed in the CGMS data. For the glycemic data arising from the CGMS devices, no statistically significant differences were observed between the two diets for any variable considered (Table 3).

\begin{tabular}{|l|c|}
\hline Sample Population & Characteristic \\
\hline Age $(\mathrm{y})$ & $62.6(9.0)$ \\
\hline Body mass $(\mathrm{kg})$ & $93.1(17.6)$ \\
\hline Height $(\mathrm{cm})$ & $167(5.0)$ \\
\hline Body Mass Index $\left(\mathrm{kg} / \mathrm{m}^{2}\right)$ & $33.4(6.6)$ \\
\hline Duration of diabetes $(\mathrm{y})$ & $8.1(8.4)$ \\
\hline Diabetes treatment regimen: & 13 \\
\hline Metformin & 3 \\
\hline Metformin and Gliclazide & 6 \\
\hline Lifestyle (non-medicated) & \\
\hline${ }^{n} \mathrm{n}=22,12$ women, 10 men & \\
\hline
\end{tabular}

Table 1: Mean (SD) characteristics of the participants*.

\begin{tabular}{|l|c|c|c|}
\hline Nutrient & Lower GI & Higher GI & P \\
\hline Glycemic index (GI) & $48(3.4)$ & $61(4.8)$ & $<0.001$ \\
\hline Glycemic load (GL) & $109(32)$ & $142(47)$ & $<0.001$ \\
\hline Energy (mJ/d) & $7.3(1.9)$ & $6.9(2.4)$ & 0.389 \\
\hline Carbohydrate (g/d) & $218(59)$ & $234(75)$ & 0.269 \\
\hline Carbohydrate (\% energy) & $49(8.5)$ & $56(10.5)$ & $<0.001$ \\
\hline Protein (\% energy) & $22(4.0)$ & $21(4.3)$ & 0.054 \\
\hline Total fat (\% energy) & $26(7.8)$ & $19(9.2)$ & $<0.001$ \\
\hline Saturated fat (\% energy) & $8(3.3)$ & $7(3.0)$ & 0.005 \\
\hline Fiber (g/d) & $32(6.2)$ & $25(6.9)$ & $<0.001$ \\
\hline
\end{tabular}

Table 2: Mean (SD) diet composition of the 22 participants from weighed diet records.

\begin{tabular}{|l|c|c|c|}
\hline Outcome measure & $\begin{array}{c}\text { Lower GI } \\
\text { (SD) }\end{array}$ & $\begin{array}{c}\text { Higher GI } \\
\text { (SD) }\end{array}$ & $\boldsymbol{P}$ \\
\hline 8-h overnight glucose (mmol/L) & $6.5(1.59)$ & $6.4(1.33)$ & 0.83 \\
\hline Mean 24 h glucose (mmol/L) & $6.62(1.49)$ & $6.31(1.27)$ & 0.31 \\
\hline Mean 3-h postprandial glucose (mmol/L) & $7.25(1.46)$ & $7.29(1.80)$ & 0.74 \\
\hline MAGE (mmol/L) & $3.67(1.35)$ & $3.90(1.44)$ & 0.61 \\
\hline Daily total AUC (mmol/L•min per 24h) & $8,906(1,810)$ & $8,786(1,666)$ & 0.82 \\
\hline Breakfast iAUC (mmol/L) & $367(273)$ & $390(279)$ & 0.69 \\
\hline Lunch iAUC (mmol/L) & $252(193)$ & $317(220)$ & 0.16 \\
\hline Dinner iAUC (mmol/L) & $216(180)$ & $263(230)$ & 0.32 \\
\hline *MAGE: Mean Amplitude of the Glycemic Excursion & & \\
\hline
\end{tabular}

Table 3: Mean (SD) glycemic outcome variables of the 18 participants for whom a complete set of continuous glucose data were obtained. 


\section{Discussion}

Our primary finding was no difference in measures of glycemia between higher and lower GI diets over four days observation in people with type 2 diabetes. The diets were well matched for energy, protein and amount of carbohydrate although the proportion of carbohydrate and fat as a percentage of energy intake, and the amount of fiber differed between diets. A lower GI diet is often associated with a higher fiber content leading to the suggestion that it is difficult to attribute independent effects on glycemia to GI or to fiber [5]. Additionally, considering different foods are used to create differences in dietary GI, differences in macronutrient content between lower and higher GI diets have been previously reported where self-selected low GI diets can result in a reduction of total carbohydrate consumed, a potential confounder to any observable GI driven effect on glycemic markers $[7,9,18]$. In our study, the lower carbohydrate and higher fat (as a percentage of energy) and the higher fiber intake on the lower GI diet would exaggerate, rather than diminish differences in postprandial glycemia. Therefore, the lack of effect of GI on our measures of glycemia is unlikely to be as a consequence of the differences in dietary composition. Both diets met the recommended acceptable macronutrient distribution range suggesting good translatability of study results. Participants with type 2 diabetes were chosen for the study due to their heightened blood glucose response to carbohydrate-containing foods $[19,20]$. As such, glycemic variability was expected to be influenced by the nature of carbohydrate given that high GI foods are characterized as inducing a higher peak glucose followed by a rapid decline compared with more gradual and less extreme fluctuations of low GI foods [4]. However this peak to trough difference, as assessed by MAGE over 48 hours, was not different between treatments $(\mathrm{P}=0.61)$.

CGMS devices have been used in dietary GI interventions in people with type 2 diabetes. For 11 patients consuming a diet with a GI some eight units lower than their habitual diet, favorable differences in mean glucose and in AUC between a single 24 hour period at baseline and another 24 hour period after seven days of eating the lower GI diet were observed [12]. A comparable result was also obtained when nine healthy young people underwent a similar protocol in which a difference in dietary GI of 7.6 units was achieved between baseline and the 7 day intervention [21]. The differences in the glycemic outcomes of these studies between baseline and the end of the intervention period were attributable to a lowering of fasting blood glucose from 8.0 to $5.3 \mathrm{mmol} / \mathrm{L}$ in the diabetic group $(\mathrm{P}<0.01)$ and from 5.4 to 4.4 $\mathrm{mmol} / \mathrm{L}$ in the young healthy group $(\mathrm{P}<0.001)$. However, neither study had a control group so that the changes in fasting blood glucose concentration could have been due to confounders. We did not find a comparable difference in fasting glucose between dietary treatments. This lack of effect on fasting glucose is consistent with other work in which fasting blood glucose appears to be relatively stable to dietary GI manipulation [22-24].

In longer-term interventions lasting several months, some effects on glycemic control in people with type 2 diabetes in response to diets differing in GI have been found together with some improvement in cardiovascular risk factors $[7,8]$. The difference in GI generated between diets in those studies was 10 GI units (glucose scale). In another trial no between-group difference was observed in glycated hemoglobin after one year when diets differing in GI by 8 units were followed [9]. Consistent with our data, total postprandial glycemic AUC was independent of the GI of meals [9]. The results of these trials might be considered underwhelming given the anticipated benefit that people with type 2 diabetes were expected to derive from choosing foods based on GI [4]. Contributing factors could be poor compliance, difficulty in generating GI differences between diets over an extended period of time, or unpredictability of glycemic responses in a whole of diet setting $[25,26]$. Food combining has been found to reduce the magnitude of the glycemic difference that is observed when individual foods are consumed [26]. The GI test is conducted under standardized laboratory conditions using a fixed amount of available carbohydrate, often in bland foods (for example: bread without spread), on subjects who remain sedentary for two hours after eating. This is not reflective of common practice in which foods are combined into meals. Additionally, physical movement of the consumer is likely to occur within two hours after eating and even modest activity has been found to reduce postprandial glycemia [27]. Hence, GI differences between foods may be optimized under GI test laboratory conditions with these differences being not fully realized in practice.

Another variable to affect acute and long-term glycemic outcome is the difference in GI units of comparative diets. We generated 13 GI units difference between diets using commonly consumed commercially available food items. It may be possible to create diets with even larger differentials in GI, although the feasibility and palatability of such changes to habitual diet is questionable with limited evidence to suggest that this may be realistically attainable over long periods [28].

A limitation of our study is the lack of a washout period between diets. This was done to enable duplicate days of CGMS recording on each diet to occur within the five working days of the week. The randomization of order in which participants consumed the diets should reduce any 'second meal effect' in which the postprandial glycemic response is influenced by the previous meal via an effect of fermentable carbohydrate on subsequent glycemia [29]. This conclusion is supported in our data by no difference in fasting glucose concentrations between dietary treatments, suggesting an absence of carryover effects. There was also a limited range of foods; it is possible that other low GI foods, such as beans, may be more effective [8].

Strengths of the study were complete dietary compliance and the excellent performance of the CGMS devices, showing good correspondence with the blood glucose readings and the expected rises in interstitial glucose levels in relation to meal consumption. Three or more peaks were observed each day, with nighttime glucose readings lower than those observed throughout the day. The lack of difference in day-long glycemia between dietary treatments is consistent with the observations that $\mathrm{HbA1C}$ is relatively resilient to dietary GI. For future research, consideration should be given to lowering not only the GI but also the amount of carbohydrate consumed. Although such a strategy of lowering the glycemic load of a diet was not effective in lowering $\mathrm{HbA1C}$, it may be that a more aggressive reduction of the glycemic load could yield measurable differences in long-term glycemic outcomes [9]. There may also be other benefits in lowering the glycemic load of diets as improvements have been found for obesity and obesity-related risks [30].

\section{Conclusions}

In our participants, food selection based on GI was not predictive of day-long glycemia. Given our previous work, and the work of others, we conclude that food combining, and perhaps movement associated with normal daily activities following meal consumption, diminishes the relative postprandial glycemic differences found when individual foods are tested under sedentary laboratory conditions $[25,26]$.

\section{Acknowledgements}

$\mathrm{BV}$ and $\mathrm{HT}$ designed and conducted the trial. AR, HT and BV undertook the 
Citation: Reynolds AN, Tekinkaya H, Venn BJ (2014) The Effect on Day-Long Glycemia of Consuming Lower and Higher Glycemic Index Diets in People with Type 2 Diabetes: A Randomized Crossover Study. J Diabetes Metab 5: 436 doi:10.4172/2155-6156.1000436

Page 5 of 5

data handling and analysis. AR prepared the manuscript, with further revision by AR and BV. The University of Otago funded the study. The authors declare no financial interest in the findings of this study.

Australia New Zealand Clinical Trials Registry trial number ACTRN12613000013763

URL http://www.anzctr.org.au

\section{References}

1. Semiz S, Dujic T, Causevic A (2013) Pharmacogenetics and personalized treatment of type 2 diabetes. Biochem Med (Zagreb) 23: 154-171.

2. Evans WE, McLeod HL (2003) Pharmacogenomics--drug disposition, drug targets, and side effects. N Engl J Med 348: 538-549.

3. Franz MJ, Bantle JP, Beebe CA, Brunzell JD, Chiasson JL, et al. (2004) Nutrition principles and recommendations in diabetes. Diabetes Care 27 Suppl 1: S36-46.

4. Jenkins DJ, Wolever TM, Taylor RH, Barker H, Fielden H, et al. (1981) Glycemic index of foods: a physiological basis for carbohydrate exchange. Am J Clin Nutr 34: $362-366$

5. American Diabetes Association (2014) Standards of medical care in diabetes--2014. Diabetes Care 37 Suppl 1: S14-80.

6. Writing Team for the Diabetes C, Complications Trial/Epidemiology of Diabetes I, Complications Research G (2002). Effect of intensive therapy on the microvascular complications of type 1 diabetes mellitus. JAMA: the journal of the American Medical Association 287: 2563-2569.

7. Jenkins DJ, Kendall CW, McKeown-Eyssen G, Josse RG, Silverberg J, et al. (2008) Effect of a low-glycemic index or a high-cereal fiber diet on type 2 diabetes: a randomized trial. JAMA 300: 2742-2753.

8. Jenkins DJ, Kendall CW, Augustin LS, Mitchell S, Sahye-Pudaruth S, et al (2012) Effect of legumes as part of a low glycemic index diet on glycemic control and cardiovascular risk factors in type 2 diabetes mellitus: a randomized controlled trial. Archives of internal medicine 172:1653-1660.

9. Wolever TM, Gibbs AL, Mehling C, Chiasson JL, Connelly PW, et al. (2008) The Canadian Trial of Carbohydrates in Diabetes (CCD), a 1-y controlled trial of low-glycemic-index dietary carbohydrate in type 2 diabetes: no effect on glycated hemoglobin but reduction in C-reactive protein. The American journal of clinical nutrition 87:114-125.

10. Klonoff DC (2005) Continuous glucose monitoring: roadmap for 21st century diabetes therapy. Diabetes Care 28: 1231-1239.

11. Praet SF, Manders RJ, Meex RC, Lieverse AG, Stehouwer CD, et al. (2006) Glycaemic instability is an underestimated problem in Type II diabetes. Clin Sci (Lond) 111: 119-126.

12. Brynes AE, Lee JL, Brighton RE, Leeds AR, Dornhorst A, et al. (2003) A low glycemic diet significantly improves the 24 -h blood glucose profile in people with type 2 diabetes, as assessed using the continuous glucose MiniMed monitor. Diabetes care 26: 548-549.

13. Atkinson FS, Foster-Powell K, Brand-Miller JC (2008) International tables of glycemic index and glycemic load values: 2008. Diabetes Care 31: 2281-2283.

14. Salmerón J, Manson JE, Stampfer MJ, Colditz GA, Wing AL, et al. (1997) Dietary fiber, glycemic load, and risk of non-insulin-dependent diabetes mellitus in women. JAMA $277: 472-477$

15. Plant and Food Research. New Zealand FOOD files 2013.

16. Service FJ, Molnar GD, Rosevear JW, Ackerman E, Gatewood LC, et al. (1970) Mean amplitude of glycemic excursions, a measure of diabetic instability. Diabetes 19: 644-655.
17. Fritzsche G, Kohnert KD, Heinke P, Vogt L, Salzsieder E (2011) The use of computer program to calculate the mean amplitude of glycemic excursions. Diabetes Technol Ther 13: 319-325

18. Gonçalves Reis CE, Dullius J (2011) Glycemic acute changes in type 2 diabetics caused by low and high glycemic index diets. Nutr Hosp 26: 546-552.

19. Trumbo P, Schlicker S, Yates AA, Poos M, Food, et al. (2002). Dietary reference intakes for energy, carbohydrate, fiber, fat, fatty acids, cholesterol, protein and amino acids. Journal of the American Dietetic Association. 102:1621-1630.

20. Ludwig DS (2002) The glycemic index: physiological mechanisms relating to obesity, diabetes, and cardiovascular disease. JAMA 287: 2414-2423.

21. Brynes AE, Adamson J, Dornhorst A, Frost GS (2005) The beneficial effect of a diet with low glycaemic index on $24 \mathrm{~h}$ glucose profiles in healthy young people as assessed by continuous glucose monitoring. $\mathrm{Br} \mathrm{J}$ Nutr 93: 179-182.

22. Brynes AE, Mark Edwards C, Ghatei MA, Dornhorst A, Morgan LM, et al. (2003) A randomised four-intervention crossover study investigating the effect of carbohydrates on daytime profiles of insulin, glucose, non-esterified fatty acids and triacylglycerols in middle-aged men. Br J Nutr 89: 207-218.

23. Philippou E, McGowan BM, Brynes AE, Dornhorst A, Leeds AR, et al. (2008) The effect of a 12-week low glycaemic index diet on heart disease risk factors and $24 \mathrm{~h}$ glycaemic response in healthy middle-aged volunteers at risk of heart disease: a pilot study. European journal of clinical nutrition 62:145-149.

24. Aston LM, Laccetti R, Mander AP, Hall R, Moore CS, et al. (2010) No difference in the 24-hour interstitial fluid glucose profile with modulations to the glycemic index of the diet. Nutrition 26: 290-295.

25. Flint A, Møller BK, Raben A, Pedersen D, Tetens I, et al. (2004) The use of glycaemic index tables to predict glycaemic index of composite breakfast meals. Br J Nutr 91: 979-989.

26. Dodd H, Williams S, Brown R, Venn B (2011) Calculating meal glycemic index by using measured and published food values compared with directly measured meal glycemic index. Am J Clin Nutr 94: 992-996.

27. Peddie MC, Bone JL, Rehrer NJ, Skeaff CM, Gray AR, et al. (2013) Breaking prolonged sitting reduces postprandial glycemia in healthy, normal-weight adults: a randomized crossover trial. The American journal of clinical nutrition 98:358-366

28. Venn BJ, Perry T, Green TJ, Skeaff CM, Aitken W, et al. (2010) The effect of increasing consumption of pulses and wholegrains in obese people: a randomized controlled trial. J Am Coll Nutr 29: 365-372.

29. Higgins JA (2012) Whole grains, legumes, and the subsequent meal effect: implications for blood glucose control and the role of fermentation. J Nutr Metab 2012: 829238.

30. Schwingshackl L, Hoffmann G (2013) Long-term effects of low glycemic index/load vs. high glycemic index/load diets on parameters of obesity and obesity-associated risks: a systematic review and meta-analysis. Nutr Metab Cardiovasc Dis 23: 699-706. 